\title{
Racetrack micro-resonators based on ridge waveguides made of porous silica.
}

\author{
P. Girault ${ }^{1}$, N. Lorrain ${ }^{1 *}$, J. Lemaître ${ }^{1}$, L. Poffo ${ }^{1}$, M. Guendouz ${ }^{1}$, I. Hardy ${ }^{2}$, M. Gadonna ${ }^{2}$,
} A. Gutierrez ${ }^{1}$, L. Bodiou ${ }^{1}$ and J. Charrier ${ }^{1}$

${ }^{1}$ UMR FOTON, CNRS, Université de Rennes 1, Enssat, F22305, Lannion, France

${ }^{2}$ UMR FOTON, CNRS, Institut Mines Telecom, Telecom Bretagne, F29238, Brest, France

* Corresponding author: nathalie.lorrain@univ-rennes1.fr

\begin{abstract}
The fabrication of micro-resonators, made from porous silica ridge waveguides by using an electrochemical etching method of silicon substrate followed by thermal oxidation and then by a standard photolithography process, is reported. The design and fabrication process are described including a study of waveguide dimensions that provide single mode propagation and calculation of the coupling ratio between a straight access waveguide and the racetrack resonator. Scanning electronic microscopy observations and optical characterizations clearly show that the microresonator based on porous silica ridge waveguides has been well implemented. This porous micro-resonator is destined to be used as an optical sensor. The porous nature of the ridge waveguide constitutes the detection medium which will enhance the sensor sensitivity compared to usual micro-resonators based on the evanescent wave detection. A theoretical sensitivity of $1170 \mathrm{~nm}$ per refractive index unit has been calculated, taking into consideration experimental data obtained from the optical characterizations.
\end{abstract}

Keywords: Porous silica; Micro-resonator; Ridge waveguides 


\section{Introduction}

Porous silica is obtained from a total oxidation of porous silicon (PS) which allows to obtain transparent porous silica with low waveguide optical losses [1, 2]. PS is easily fabricated by electrochemical [3] etching of silicon. The tunability of the porosity and of the thickness of PS layers which depends on fabrication parameters, allows optoelectronic components [4], filters [5,6], sensors for chemical [7, 8], biological $[9,10,11,12,13]$ applications to be developed. Indeed, the large internal surface of PS and its biocompatibility are significant advantages for biosensing applications [11]. However, as the surface of PS is strongly hydrophobic, thermal oxidation and chemical adaptation must be applied respectively to stabilize and functionalize the internal surface of the porous silicon layer to obtain subsequent efficient molecules attachment. The oxidation of PS has been extensively investigated by several authors in order to fabricate SOI structures or to form a dielectric isolation material for IC components [14] or integrated optical waveguides [15].

The partially or totally oxidized PS material can be used as a host for various molecules which can either be in solution in the pores, or grafted onto the internal surface after the functionalization of the PS [10]. In most cases, these molecules can be detected by a change in the refractive index of the porous material, induced by their presence inside the pores. Indeed, the refractive index is a weighted average of the refractive index of silicon (if partial oxidation), silica, and the components inside the pores (molecules, air...). For small sized pores (less than the optical sensing wavelength) the effective medium method can be used to estimate the refractive index of the PS, which can be even partially or totally oxidized (porous silica), and the volume fraction of the different constituents [16-17]. 
Optical sensors based on PS using refractive index variations [11] have already been the subject of several studies, the number of which continues to grow due to the interest of this material for increasing the sensor sensitivity. Among components based on PS partially or totally oxidized, microcavities sensors [9,17,18], Bragg reflectors [8] and planar ARROWs [19] do not need any photolithographic steps for fabrication as only vertical light confinement is required. Other optical structures have been developed with both lateral and vertical confinement using photolithography processes [20,21] or a direct laser writing method [22]; for example, buried waveguides have been used to fabricate integrated Mach-Zehnder interferometers for sensing applications [23, 24]. Recently, the first single side coupled micro-ring resonator based on PS [25] has been fabricated using standard electron beam lithography and reactive ion etching. The porous core ridge waveguide height was $600 \mathrm{~nm}$. The highest sensitivities of optical sensors based on PS reported to date are obtained from spectral measurements of resonant cavities [26] (300-500 nm per Refractive Index Unit (RIU)) or of grating coupled waveguides (1000 nm/RIU) [12] made using electron beam lithography and reactive ion etching for the detection of peptide nucleic acid (PNA).

Micro-Resonators (MRs) for sensing applications are now being widely investigated as they offer the advantage of significant miniaturization of the device, which greatly reduces the number of molecules needed for detection [27, 28].

The best $\mathrm{Q}$ factors, better than $10^{7}$, are obtained with microsphere resonators using whispering-gallery mode (WGM) resonances [29, 30]. The WGM have a large potential for sensing application with very low optical detection limits as they are able to react to a monolayer of molecules adsorption [29,31]. 
However, microsphere resonators lack integration capability [32], which limit their use in practical applications. To solve these problems, integrated micro-ring, racetrack or micro-disk resonators are used, albeit with reduced Q factors [33]. In integrated optics, the Q factor is limited in the range of $10^{4}-10^{5}$ because of the optical losses into the resonators due mainly to the material and the technological process.

Silicon and polymers are the main materials used in the fabrication of integrated resonant structures, which rely on the evanescent waves to detect the presence of molecules whether adsorbed on the sensor surface (surface sensing) or spread into the surrounding medium (homogeneous sensing) [34].

However, the sensitivity of integrated MRs can be improved by optimizing the interaction between the molecules and the optical wave. This optimization can be provided by the use of a porous waveguide core that will allow a direct interaction between the propagated light and the molecules to be detected [12]. Mancuso et al. [35] have used a nanoporous polymer for waveguide MRs cores, allowing an increase in the device sensitivity of $40 \%$.

In the present work, fully oxidized PS (or porous silica) will be used for the core of the MR waveguides so as to greatly enhance the interaction between the propagated light and detected molecules and thus the sensitivity. The first all PS single side coupled micro-ring [25] with ring radii of 10 or $25 \mu \mathrm{m}$ and a gap between the ring and the straight waveguides of $200 \mathrm{~nm}$, gave a detection sensitivity of $380 \mathrm{~nm} / \mathrm{RIU}$ when salt water solutions infiltrated the device. Besides, a theoretical study of a PS micro-ring resonator with a rib waveguide geometry has been performed by Hutter et al [36]. Their study clearly demonstrated the potential of a PS micro-ring for sensing application with a single ring resonator. An improved sensitivity of $700 \mathrm{~nm} / \mathrm{RIU}$ was found in their simulations compared to $380 \mathrm{~nm} / \mathrm{RIU}$ for a conventional micro-ring with the same 
design and a detection mode based on evanescent waves. Higher values could be reached by using a cascade of micro-resonators [37] or by an integration of micro-resonators into a Mach Zehnder interferometer [38].

For the conception of a MR, low loss waveguides supporting single-mode propagation are required [28]. The design can be horizontal, the most widely used because its simplicity, or vertical, which requires several photolithographic mask levels [39]. For a horizontal design, lateral flanks of the core waveguides have to present a rectangular section in the coupling region for the control of the coupling ratio between a straight access waveguide and a resonator waveguide. Up to now, porous silica buried single mode waveguides have been fabricated with losses from about $1 \mathrm{~dB} / \mathrm{cm}$ [22] to $4 \mathrm{~dB} / \mathrm{cm}$ [40] but the lateral flanks were not vertical but were slightly rounded.

To date, no rib based on partially or totally oxidized PS single mode waveguide with vertical lateral flanks and with a height larger than $1 \mu \mathrm{m}$ has been fabricated.

In this study we demonstrate the feasibility of the fabrication of a racetrack MR based on porous silica ridge waveguides using standard photolithographic patterning with a polymer photoresist. In the first part, we present the fabrication method and the characteristics of the porous silica layers obtained. Then the photolithography method implemented to elaborate the racetrack micro-resonator based on ridge waveguides made of porous silica is described. In a second part, we detail calculation methods that we have implemented to get the waveguide dimensions required for single mode propagation and to determine the coupling ratio between the straight and the racetrack ridge waveguides. Then, the design of the MRs based on ridge waveguides made of porous silica is detailed including the waveguide dimensions for single mode propagation and the results of the coupling study for a given range of gap. MRs obtained 
are structurally and optically characterized. As the MRs will be used for sensing application, we also present a preliminary theoretical study of the porous MRs sensitivity.

\section{Material and Implementation method}

\subsection{Porous silica layers}

Three consecutive PS layers were prepared by electrochemical anodization of a heavily doped P substrate with three successive current densities of 4, 50 and $100 \mathrm{~mA} / \mathrm{cm}^{2}$ for specific times. The electrolyte was composed of $(50 \%) \mathrm{HF}$, ethanol and deionized water solution with ratios of 2:2:1 respectively. Following this, the PS layers were completely oxidized at $960{ }^{\circ} \mathrm{C}$ for 1 hour under a humid oxygen flow in order to obtain porous silica layers. The first porous (upper) layer $\left(4 \mathrm{~mA} / \mathrm{cm}^{2}\right)$ presents a low porosity (around $\left.6 \%\right)$ and plays the role of a "technological barrier" that prevents the other deeper porous silica layers from being infiltrated by the resin during the subsequent photolithography process which is necessary to create the ridge porous waveguides. The second $\left(50 \mathrm{~mA} / \mathrm{cm}^{2}\right)$ and the third $\left(100 \mathrm{~mA} / \mathrm{cm}^{2}\right)$ porous layers constitute the core and the lower cladding layers respectively of the porous silica waveguides.

The porosity and the refractive index of the core and cladding silica porous layers were extracted using the Bruggeman model from reflectivity spectra of two single porous layers whose fabrication parameters corresponds to the core and the cladding respectively [2, 17]. The accuracy of $60 \mathrm{~nm}$ on the thicknesses of the single porous layers induces errors of $5 \%$ and 0.02 RIU on the porosity and on the refractive index respectively. 
The values of porosities and refractive indices of the core and the cladding porous silica layers are given in Table I. We also have mentioned the porosity of the porous silicon layers before their full oxidation.

Table I: Porosity of the PS layers before oxidation and photolithography steps. Porosity and refractive index at $1550 \mathrm{~nm}$ for the porous silica ridge waveguide structure.

\begin{tabular}{|c|c|c||}
\hline \hline Porous layers & Core & Cladding \\
\hline \hline Porosity (\%) before oxidation & 62 & 71 \\
\hline \hline Porosity (\%) after full oxidation & 32 & 50 \\
\hline \hline Refractive index & 1.30 & 1.22 \\
\hline
\end{tabular}

As in ref [2], the adjustment of theoretical and experimental reflectance spectra is obtained by considering a total oxidation of the porous silicon layers. Moreover, the use of the M-lines method have also shown the same indices taking into consideration the accuracies of measurements. Besides, as mentioned in reference [14, 41], as the porosities of the core and the cladding layers are, before the oxidation step, higher than $56 \%$ (table I), a full oxidation of PS is obtained. However, a slight difference in the stoechiometry compared to silica could occur [42], Beside, according to Yakovtseva et al [15], with the use of a temperature of $800-900^{\circ} \mathrm{C}$, the quality of the material obtained is still different from that a standard thermal $\mathrm{SiO}_{2}$.

Yet, as the effect on the change in the indices is lower than the accuracies of the measures, we have considered that our porous silicon layers are totally transformed into porous silica. 


\subsection{Photolithography process}

The ridge porous waveguides were then created using a standard photolithography process on the porous silica layers. The process is described for a straight waveguide in Fig. 1. A positive SPR photosensitive resin layer was deposited by spin coating on the top of the structure. Patterns were then produced under UV exposure through a well-defined chrome mask designed using Olympios software.

To obtain the desired ridge waveguides, a trifluoromethane $\left(\mathrm{CHF}_{3}\right)$ plasma $\mathrm{RIE}$ (Reactive Ion Etching was performed. Carefully checking was required to be sure that the resin had not infiltrated the pores through the first low porous layer (barrier porous silica layer) during the photolithography process. In order to verify this step, the method used relies on reflectivity measurements of a single porous layer obtained with the same anodization parameters (4 $\mathrm{mA} / \mathrm{cm}^{2}$ ) as the porous barrier layer, without and with the resin deposit.

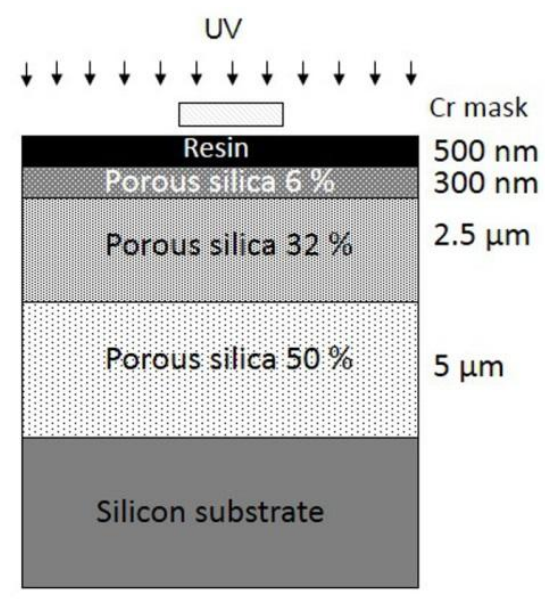

(a)

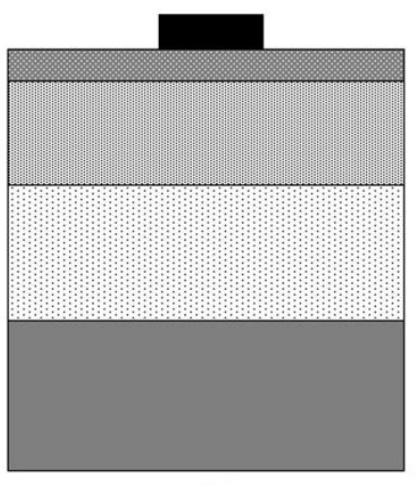

(b)

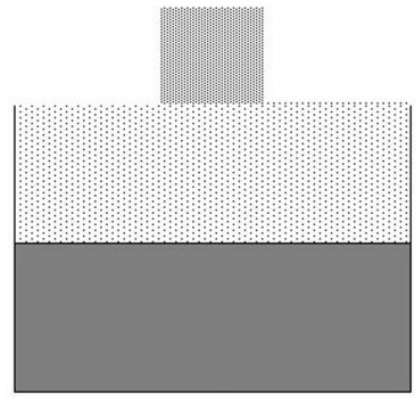

(c)

Fig. 1. Schema of the photolithography process used for the fabrication of porous silica ridge waveguides: a) deposit of a SPR photosensitive resin and irradiation under UV 
exposure; b) wet etching development of the photosensitive resin; c) dry etching of the porous silica layers ("technological barrier" and core) under the unprotected area.

To obtain a good resolution, the thickness of the photosensitive resin has to be the thinnest. Yet, it has to be thick enough in order to allow the etching of the core layer before the complete etching of the photosensitive resin without which the patterns are not well defined. The selectivity, the ratio between the etching rate of the photosensitive resin and the core layer (shown in table II), has to be the highest. By adjusting RIE parameters, we experimentally obtained a selectivity around 5. Then, knowing the selectivity, we have to adjust the resin thickness in order to get a target core thickness at the end of the process.

Table II: RIE dry etching rate for the different layers

\begin{tabular}{|c|c|c|c||}
\hline $\begin{array}{c}\text { Layers in the } \\
\text { photolithography } \\
\text { process }\end{array}$ & Resin SPR & $\begin{array}{c}\text { Porous silica } \\
\text { core }\end{array}$ & $\begin{array}{c}\text { Porous silica } \\
\text { cladding }\end{array}$ \\
\hline $\begin{array}{c}\text { Photolithography } \\
\text { etching rate }(\mathrm{nm} / \mathrm{s})\end{array}$ & 50 & 250 & 500 \\
\hline
\end{tabular}

\section{Calculation methods}

\subsection{Waveguide dimension range for single mode propagation}

The dimensions of the porous silica core waveguide have to be chosen to provide single mode propagation at $1.55 \mu \mathrm{m}$. The effective refractive index method [43] and Matlab software were used to calculate the effective refractive index of the ridge porous silica waveguide as a function of its width and height for three different TE and TM modes: the 00 fundamental 
mode, the 01 mode and the 10 mode. Then, the lowest dimension limits (width and height) that involve solutions to the Maxwell equations [43] for these TE and TM modes propagation, were deduced. The possible dimensions of the ridge waveguides required to obtain only single mode (00 mode) propagation for both the TE and TM modes, are between the lower limit dimensions of the 00 mode and the lower limit dimensions of the 01 and the 10 modes.

\subsection{Calculations of the coupling ratio between access and micro-resonator waveguides}

The MR configuration which has been studied is a single side coupled racetrack resonator. Fig. 2.a represents the top view of the coupling area that has been considered and which includes the straight waveguide and half of a racetrack waveguide.

The separation (gap) "g" between the straight waveguide and the MR is an important parameter which contributes to the coupling ratio. In order to calculate the coupling ratio for a range of gaps studied, we have used the method described in the following for both the TE and TM modes.

Fig. 2.b is a schematic cross section which shows the straight and the ring core waveguides (refractive index $\mathrm{n}_{1}$ ) surrounded by the lower (refractive index $\mathrm{n}_{2}$ ) and upper cladding (refractive index $\mathrm{n}_{3}$ ) layers at the minimal gap "g".

In this configuration, the gap between the straight and the racetrack waveguide is a constant in the $\mathrm{z}$ axis only along the straight coupling area (distance $\mathrm{L}_{\mathrm{c}}$ ).

In the y direction, in the curved coupling area, the distance separation between the straight and the racetrack waveguides, as a function of $\mathrm{z}$, can be expressed by the following equation:

$$
y(z)=g+R_{e}(1-\cos \theta)
$$


where $\mathrm{g}$ is the minimal distance between the straight and the racetrack waveguides, $\mathrm{R}_{\mathrm{e}}$ is the extern radius and $\theta$, defined by $\theta=\arcsin \frac{\mathrm{Z}}{\mathrm{R}_{\mathrm{e}}}$, varies from 0 to $\frac{\pi}{2}$.

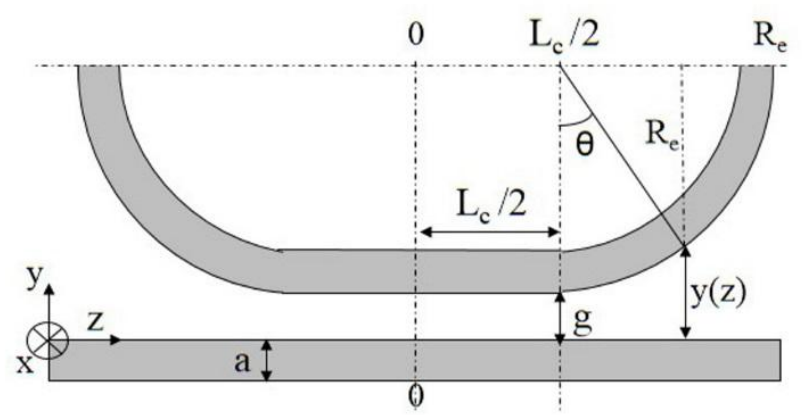

(a)

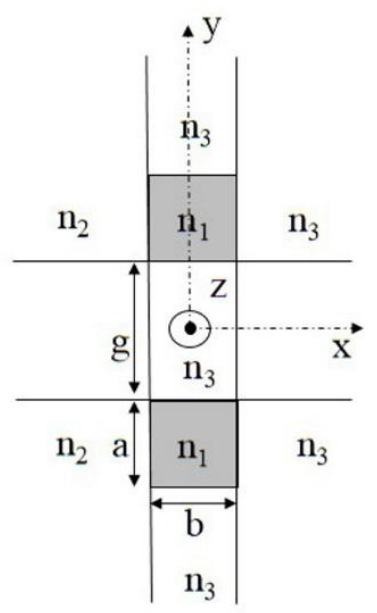

(b)

Fig. 2. Schema of the coupling region of racetrack resonator: (a) top view and (b) cross section view at $\mathrm{z}=0$.

We note that $\mathrm{C}_{\mathrm{T}}(\mathrm{z})$ the coupling coefficient for a $\mathrm{z}$ abscissa, given in the Marcatili method [44] (for the TE and TM modes) is defined by: 


$$
\left|C_{T}(z)\right|=2\left(\frac{\mathrm{k}_{\mathrm{x}} \mathrm{A}_{3}}{\pi}\right)^{2} \frac{\mathrm{k}_{1}}{\mathrm{k}_{\mathrm{z}}} \frac{\left[1-\left(\frac{\mathrm{n}_{3}}{\mathrm{n}_{1}}\right)^{2}\right]^{\frac{1}{2}}}{\mathrm{a}}\left[1-\left(\frac{\mathrm{k}_{\mathrm{x}} \mathrm{A}_{3}}{\pi}\right)^{2}\right]^{\frac{1}{2}} \exp \left\{-\pi \frac{\mathrm{y}(\mathrm{z})}{\mathrm{A}_{3}}\left[1-\left(\frac{\mathrm{k}_{\mathrm{x}} \mathrm{A}_{3}}{\pi}\right)^{2}\right]^{\frac{1}{2}}\right\}
$$

where:

- $\quad \mathrm{a}$ is the core waveguide width (in the y transverse direction),

- $\mathrm{k}_{\mathrm{z}}$ is the axial propagation constant which depends on the transverse propagation constants $\mathrm{k}_{\mathrm{x}}$ and $\mathrm{k}_{\mathrm{y}}$, and on the propagation constant $\mathrm{k}_{1}$ in the free space medium of refractive index $\mathrm{n}_{1}$; the constant $\mathrm{k}_{\mathrm{z}}, \mathrm{k}_{\mathrm{x}}$ and $\mathrm{k}_{\mathrm{y}}$ for the TE and TM modes can be expressed by:

$$
\begin{aligned}
& \mathrm{k}_{\mathrm{z}}=\left(\mathrm{k}_{1}^{2}-\mathrm{k}_{\mathrm{x}}^{2}-\mathrm{k}_{\mathrm{y}}^{2}\right)^{\frac{1}{2}} \\
& \mathrm{k}_{\mathrm{x}}=\frac{\pi}{\mathrm{a}}\left(1+\frac{2 \mathrm{n}_{3}^{2} \mathrm{~A}_{3}}{\pi \mathrm{n}_{1}^{2} \mathrm{a}}\right)^{-1} \\
& \mathrm{k}_{\mathrm{y}}=\frac{\pi}{\mathrm{b}}\left(1+\frac{\mathrm{A}_{2}+\mathrm{A}_{3}}{\pi \mathrm{b}}\right)^{-1} \\
& \mathrm{k}_{\mathrm{x}}=\frac{\pi}{\mathrm{a}}\left(1+\frac{2 \mathrm{~A}_{3}}{\pi \mathrm{a}}\right)^{-1} \\
& \mathrm{k}_{\mathrm{y}}=\frac{\pi}{\mathrm{b}}\left(1+\frac{\mathrm{n}_{3}^{2} \mathrm{~A}_{2}+\mathrm{n}_{3}^{2} \mathrm{~A}_{3}}{\pi n_{1}^{2} \mathrm{~b}}\right)^{-1}
\end{aligned}
$$

The transverse propagation constants $\mathrm{k}_{\mathrm{x}}$ and $\mathrm{k}_{\mathrm{y}}$ depend on the refractive indices (Fig. 2.b) of the core $\left(\mathrm{n}_{1}\right)$, the lower $\left(\mathrm{n}_{2}\right)$ and the upper cladding $\left(\mathrm{n}_{3}\right)$ layers of the waveguides and on the coefficients $A_{i}$ which are defined for $i=2,3$ by the following expression : 


$$
A_{i}=\frac{\pi}{\left(\mathrm{k}_{1}^{2}-\mathrm{k}_{\mathrm{i}}^{2}\right)^{\frac{1}{2}}}
$$

Then to obtain the coupling coefficient $\mathrm{C}_{\mathrm{w}}$ over the whole distance of the coupling area (Fig.

2.a), the $\mathrm{C}_{\mathrm{T}}(\mathrm{z})$ function has to be integrated between $-\frac{L c}{2}$ and $\mathrm{R}_{\mathrm{e}}$ and then multiplied by a factor 2 because of the symmetry of the geometry:

$$
\mathrm{C}_{\mathrm{w}}=2\left(\int_{\frac{L_{c}}{2}}^{\mathrm{R}_{\mathrm{e}}} \mathrm{C}_{\mathrm{T}}(\mathrm{z}) \mathrm{dz}+\mathrm{C}_{\mathrm{T}}(\mathrm{z}) \times\left(\frac{\mathrm{L}_{\mathrm{c}}}{2}\right)\right)
$$

Then the coupling ratio $\kappa^{2}$ is equal to [43]:

$$
\kappa^{2}=1-\left(\cos \left(C_{W}\right)\right)^{2}
$$

\section{Results and discussion}

\subsection{Design of the all porous silica micro-resonator}

\subsubsection{Waveguide dimension range for single mode propagation}

Using the method described in the part 3.1, the lowest dimension limits (width and height) that involve solutions to the Maxwell equations [43] for the $\mathrm{TE}_{00}, \mathrm{TE}_{01}$ and $\mathrm{TE}_{10}$ modes propagation were calculated and plotted in Fig. 3. 


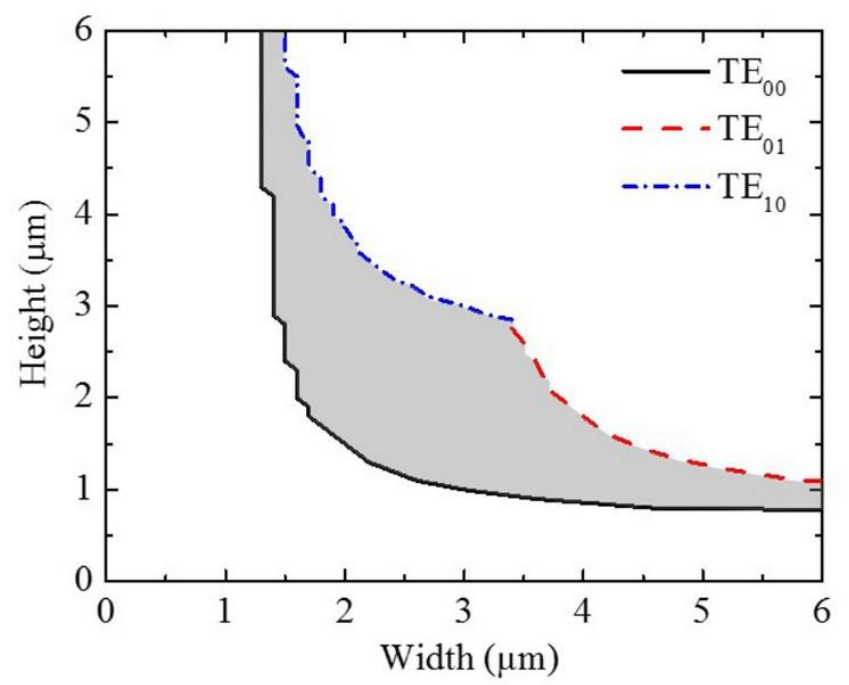

Fig. 3. Solutions of the Maxwell equations for the $\mathrm{TE}_{00}, \mathrm{TE}_{01}$ and $\mathrm{TE}_{10}$ modes as a function of the porous silica ridge waveguide dimensions. The grey area between the $\mathrm{TE}_{00}$ fundamental mode curve and the $\mathrm{TE}_{01}$ and $\mathrm{TE}_{10}$ modes curves represents the waveguide dimensions range for which only single mode propagation is possible.

The grey area between the lowest limits of the $\mathrm{TE}_{00}$ mode (fundamental mode) and the $\mathrm{TE}_{10}$ and $\mathrm{TE}_{01}$ modes, represents the single mode region. For $\mathrm{TM}$ modes, the curves are similar to $\mathrm{TE}$ modes but are slightly shifted towards higher dimension values. Based on these results, the waveguide width could be between $1.5 \mu \mathrm{m}$ and $3.5 \mu \mathrm{m}$ so that a wide range of height (typically from about $1 \mu \mathrm{m}$ to $3 \mu \mathrm{m}$ ) could allow single mode propagation. In this dimension range, we have chosen to experimentally target a waveguide width of $2 \mu \mathrm{m}$ and a height waveguide of 2.5 $\mu \mathrm{m}$. Then, for these chosen dimensions, an effective refractive index of $1.238 \pm 0.020$ for the TE mode and $1.243 \pm 0.020$ for the TM mode were calculated using the effective index method [43].

Similar values were obtained from the Finite Difference Generic (FDG) method which is a full vectorial solver from Olympios simulation software. The accuracies on the calculated 
refractive indices take into account the measurements errors on the refractive index of the core and cladding porous silica layers.

\subsubsection{Coupling study between the access and the porous micro-resonator waveguides}

Since the porous silica MR will be used for sensing applications, the MR is designed as a racetrack with a radius $\mathrm{R}$ of $25 \mu \mathrm{m}$ in order to obtain a free spectral range superior to $5 \mathrm{~nm}$ which is sufficient enough to get a large detection window. Furthermore, to obtain a sufficient coupling ratio between the straight and the curved waveguides, different coupling length $\mathrm{L}_{\mathrm{c}}$ have been studied $(0,30,40,50,60$ and $70 \mu \mathrm{m})$. Considering that the theoretical resolution limit of photolithography is $0.4 \mu \mathrm{m}$ for polymer materials [45], gaps varying from 0.5 to $1 \mu \mathrm{m}$ have been studied.

Using the calculation method defined in part 3.2, the coupling ratio $\kappa^{2}$ has been calculated at $\lambda=1550 \mathrm{~nm}$ as a function of the gap "g" for a waveguide width of $2 \mu \mathrm{m}$ and a height of $2.5 \mu \mathrm{m}$ for different studied coupling lengths $\mathrm{L}_{\mathrm{c}}$.

For a coupling length of $\mathrm{L}_{\mathrm{c}}=70 \mu \mathrm{m}$ and within the studied gap range $(0.5 \mu \mathrm{m}$ to $1 \mu \mathrm{m})$ that can be obtained from a process point of view, the coupling ratio varies from $8 \%$ to $90 \%$ for TE mode and from $2 \%$ to nearly $70 \%$ for TM mode (Fig. 4). 


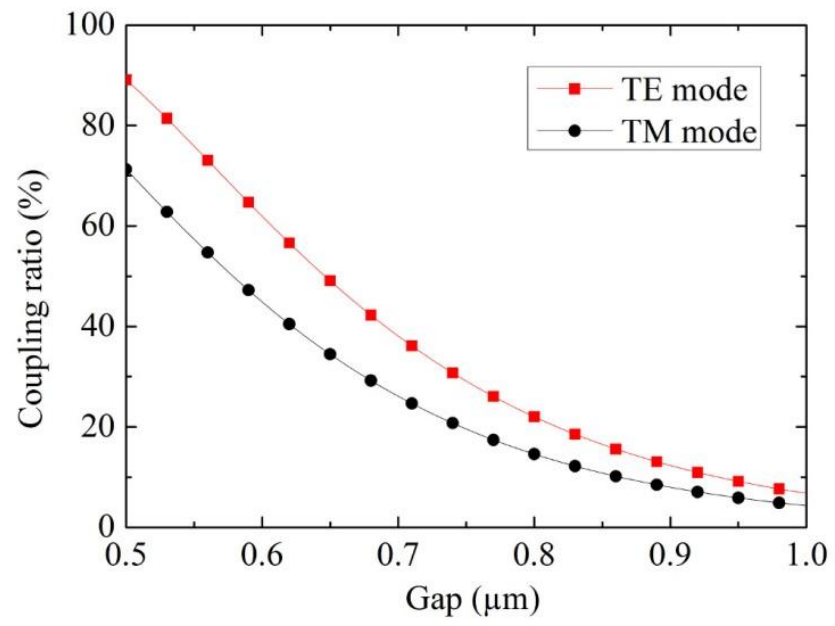

Fig. 4. Calculated coupling ratio as a function of the gap between the straight and the curved waveguides for the TE and TM modes for a waveguide width of $2 \mu \mathrm{m}$ at $1550 \mathrm{~nm}$ $\left(\mathrm{R}=25 \mu \mathrm{m}\right.$ and $\left.\mathrm{L}_{\mathrm{c}}=70 \mu \mathrm{m}\right)$.

For this case, the range of gap studied here allows us to reach a wide scale of coupling ratios that should be sufficient to obtain good MR transmission spectra.

In Fig. 5, the top view (a) and the cross section (b) of the porous silica racetrack MR design are reported with the chosen waveguide dimensions and the MR geometry that provide a sufficient coupling ratio $\left(\mathrm{L}_{\mathrm{c}}=70 \mu \mathrm{m}\right)$ and a sufficient free spectral range which is in this case of $6.5 \mathrm{~nm}$ for future sensing applications. 
(a)

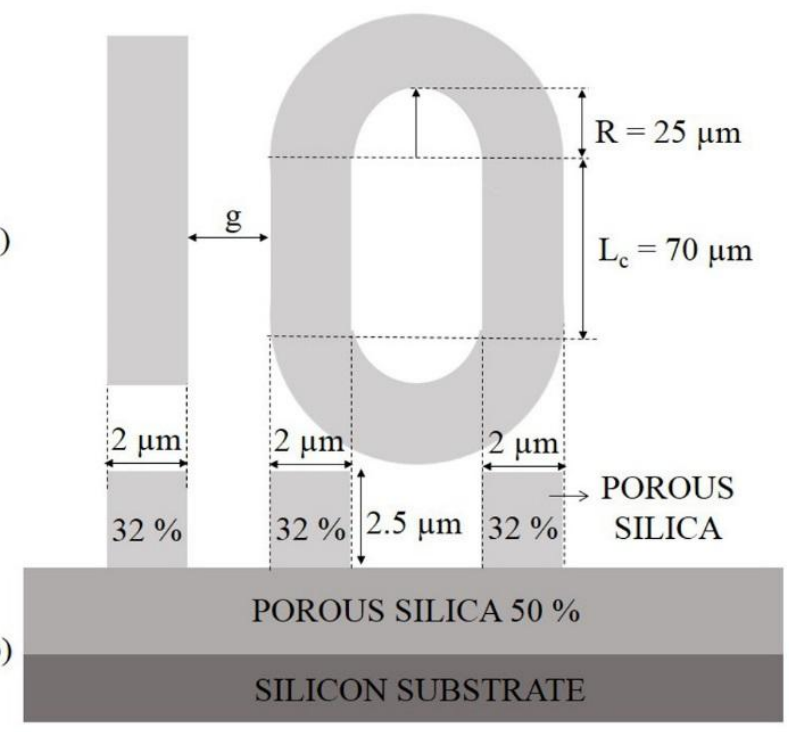

Fig. 5. Schema of a top view (a) and a cross section (b) of the porous silica racetrack MR.

\subsection{Control of manufacturing process}

Scanning Electronic Microscopy (SEM) images of the ridge racetrack porous MR are presented in Fig. 6. The cross section of the etched waveguide in Fig. 6.a shows a waveguide core, the height of which is $2.5 \pm 0.1 \mu \mathrm{m}$. The core is rectangular only in its lower half, with a width of $1.8 \mu \mathrm{m}$ instead of the $2 \mu \mathrm{m}$ expected from the photolithography mask. During the photolithographic step of etching the top of the porous layers, the edges of the ridge waveguides are also affected, more so in the top half, which explains the width profile.

The SEM top view of the whole structure is given in Fig. 6.b. At this magnification, the racetrack is well defined and with the target dimensions. The separation between the straight and the racetrack MR ridge, constituting the coupling region, has been well achieved. At a high magnification of the coupling region (Fig. 6.c and Fig. 6.d), the porous core waveguide with a 
width of $1.8 \mu \mathrm{m} \pm 0.1 \mu \mathrm{m}$, appears in light grey whereas the cladding layer shows as dark grey in the SEM image.

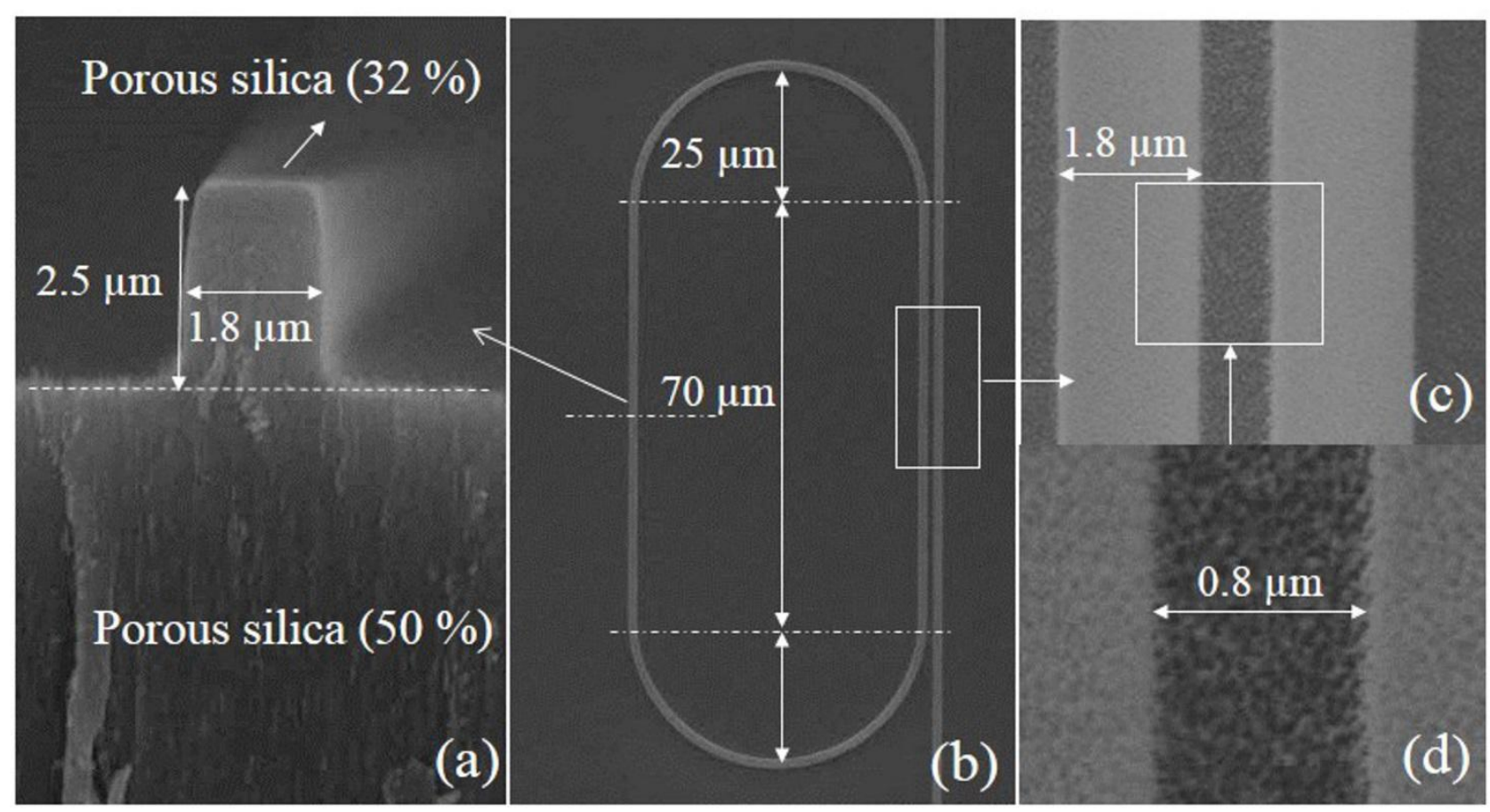

Fig. 6. SEM images of the racetrack resonator: (a) cross section of a porous silica waveguide; top views at different scales: (b) whole racetrack MR, (c) the two waveguides in the coupling area, (d) the gap separation between the two waveguides of the coupling area.

In Fig. 6.d, the pores are visible and have larger dimensions in the cladding layer than in the core layer, in accordance with their respective porosities. The gap between the straight waveguide and the racetrack waveguide is $0.8 \mu \mathrm{m} \pm 0.1 \mu \mathrm{m}$ instead of the $0.6 \mu \mathrm{m}$ expected. The real separation distance between the two waveguides is higher than expected by $0.2 \mu \mathrm{m}$ because of the additional lateral etch that occurred at the top of the porous layers. 


\subsection{Transmission responses of the porous silica racetrack MR}

In order to verify that the porous silica racetrack MR is optically operational, the spectral transmission responses, for different gaps, were measured. The output from a tunable wavelength laser is injected in the input straight waveguide using a lensed fiber with a mode radius of 2.2 $\mu \mathrm{m}$. A second lensed single mode fiber is also used to couple the output of the straight waveguide to a power meter or to an optical spectrum analyzer. A polarization controler was inserted between the output of source and the first lensed fiber. The transmission measurements were done with a step resolution of $1 \mathrm{pm}$ over the wavelength range of $1545-1560 \mathrm{~nm}$ at the output of the straight waveguide. The measured transmission as a function of the wavelength for one polarization of light is reported in Fig. 7 for the experimtental gap of $0.8 \mu \mathrm{m}$. The spectrum shows two resonance wavelengths at $1549 \mathrm{~nm}$ and $1555 \mathrm{~nm}$ in the range studied. This demonstrates that the light is propagated in the waveguide and in addition, good coupling of the light occurs between the access waveguide and the MR. This experiment shows clearly that we have succeed in making a ridge porous silica racetrack MR.

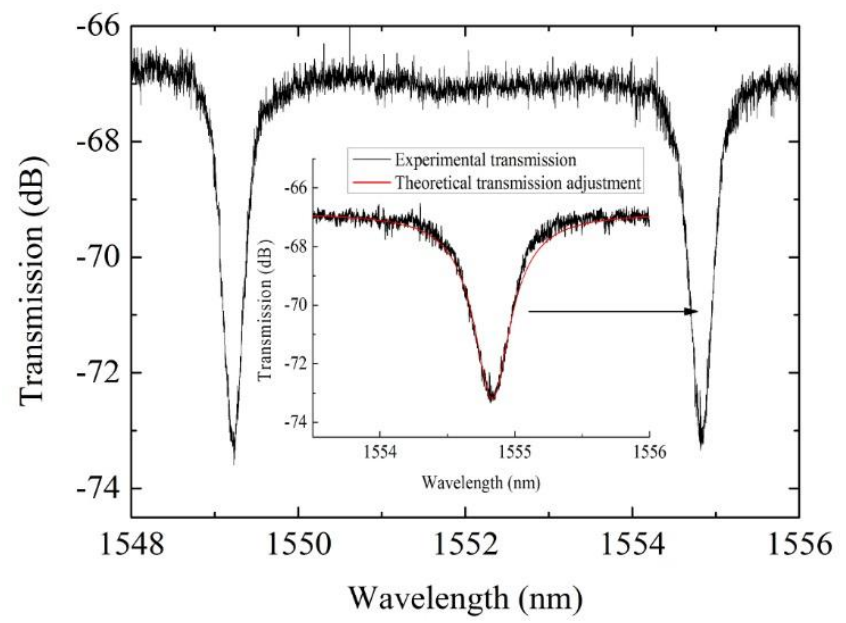

Fig.7. Experimental transmission spectra for one polarization of light. In the insert, the theoretical transmission adjusted to the experimental one is shown. 
From the experimental transmission spectra (Fig. 7), the following characteristics of the resonator that we have already described in reference [46] have been deduced, with the same assumption that the coupling losses are negligible : an extinction ratio of around $6.5 \mathrm{~dB}$, a Free Spectral Range (FSR) of $5.6 \mathrm{~nm}$ which is of the order of magnitude expected, taking into account the geometry of the design and the effective index. A full width at half maximum $(\delta \lambda)$ of 0.45 $\mathrm{nm}$ is obtained, with a corresponding quality factor $\mathrm{Q}$ of 3392 .

Using the equations defined in our previous study on polymer micro-ring resonators [47], the theoretical transmission has been calculated and then adjusted to match the experimental one. The adjustment of the resonance shape (insert in Fig. 7) is performed by modifying the coupling ratio and the propagation losses in the ring, whereas the FSR is adjusted with the group index parameter. A coupling ratio $\kappa^{2}$ of $32.5 \pm 1.0 \%$ and a field attenuation constant $\mathrm{a}=0.931$, which corresponds to propagation losses per length unit $(\alpha)$ of $20.4 \pm 0.4 \mathrm{~dB} / \mathrm{cm}$, have been obtained in this way. To match the experimental FSR, the group index $n_{\mathrm{g}}$ was adjusted to $1.4175 \pm 0.0001$.

Due to the width of waveguides being slightly smaller than expected, it is difficult to compare the experimental coupling ratio to the theoretical one obtained previously from the coupling study that was performed for a waveguide width of $2 \mu \mathrm{m}$ (Fig. 4). Therefore, using this same method, the coupling ratio, over the gap range from $0.7 \mu \mathrm{m}$ to $0.9 \mu \mathrm{m}$ for a waveguide width of $1.8 \mu \mathrm{m}$ corresponding to the one measured on the SEM image (Fig. 6.d), has been recalculated for the TE mode. The experimental value of $32.5 \%$ corresponds to a gap of $0.82 \mu \mathrm{m}$ for the TE mode and to a gap of $0.74 \mu \mathrm{m}$ for the TM mode. The gap value of the $0.82 \mu \mathrm{m}$ for the TE mode is very close to of the experimental one, meaning that this is the mode of polarization that has been selected. 


\subsection{Theoretical sensing study}

In order to use the porous silica MR for biosensing application, an activation of the surface is needed. In a previous study [48], the functionalization of single porous silica layer for Bovin Serum Albumin (BSA) attachment has been studied. The functionalization procedure including a silanization step 3 using Aminopropyltriethoxysilane (APTES) and a coupling step using Glutaraldehyde (GL) molecules, has been optimized using Raman spectroscopic and Fourier Transform Infrared Spectroscopy (FTIR). A modelling study of the reflectance spectra and the use of the Bruggemann model allowed the volume fraction and refractive index variations to be estimated after each step by assigning them with the number of molecules of APTES, GL and BSA grafted on the porous silica.

The active porous silica layer used in the porous silica MR is prepared in the same conditions as in reference [48]. So in order to estimate the sensitivity and the detection limit, we have taken into consideration the refractive index before the BSA grafting, equal to 1.369 [48] for the calculation of the MR transmission spectra.

We have supposed that the refractive index of the waveguide lower cladding layer are not affected by the functionalization process. This hypothesis can be experimentaly taken into consideration by adding a technological barrier to prevent from the molecules inflitration in the lower cladding. We also have neglected the change of losses that likely can occur, as the losses do not affect the resonant wavelenght. Then, we have calculated the transmission spectra for different volume fractions of BSA grafted on the functionnalized porous silica. The volume fraction of BSA is correlated to the number of molecules grafted per surface unit on the functionalized porous silica, number which has been estimated taking into consideration the 
surface specific area of $30 \mathrm{~m}^{2} / \mathrm{cm}^{3}$ calculated from [49], the volume of BSA of $125 \mathrm{~nm}^{3}$ [50] and the molar weight of $66.43 \mathrm{kDa}$ of BSA [50]. Figure 8 represents the resonant shift calculated as a function of the refractive index variation and as a function of the correlated volume fraction of grafted BSA molecules in the range of the FSR.

The theoretical and expected sensitivity of the porous silica MR reaches $1170 \mathrm{~nm} / \mathrm{RIU}$ (Refractive Index Unit) which corresponds to a sensitivity of $0.02 \mathrm{~nm} / \mathrm{pg} / \mathrm{mm}^{2}$. Such a sensitivity value is high for such single porous silica MR and is very promising for the sensing applications.

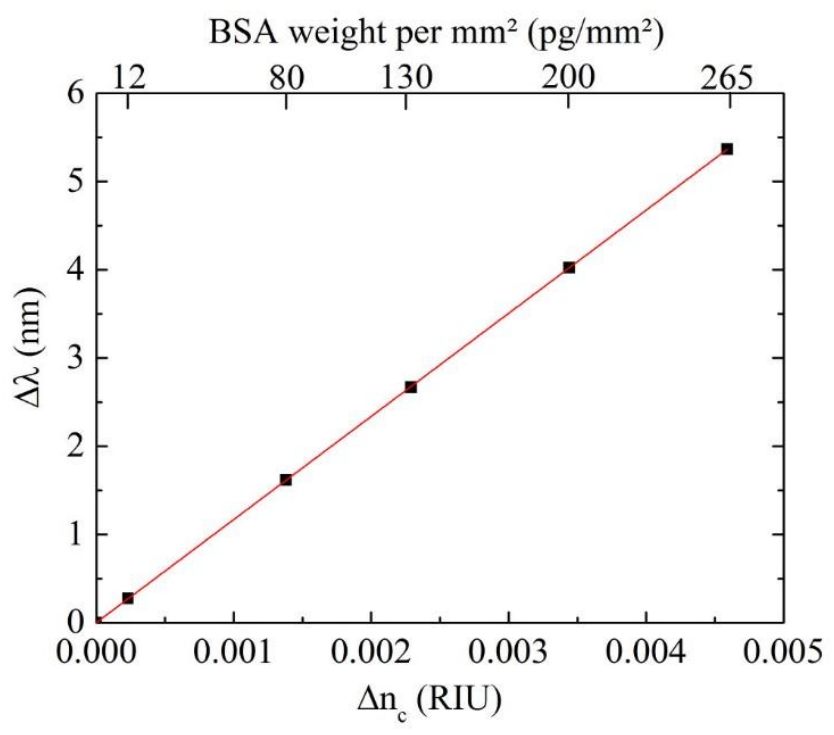

Fig.8. Resonant wavelenght shift deduced from calculated transmission spectra of the functionalized porous silica racetrack after BSA grafting, resonator taking into account the adjustment parameters obtained from Fig. 7.

We also have estimated the detection limit taking into consideration the minimal shift that is detectable with the same characteristics of MRs obtained from the experimental MRs optical 
characterizations. At this stage, we thus supposed that the functionalization does not affect the quality factor of the MRs. However, it is reported that the activation of the surface of MRs such as for example microspheres which have been functionalized for specific recognition of proteins induced a spoiling of the quality factor [30].

A detection limit of $10 \mathrm{pg} / \mathrm{mm}^{2}$ has been estimated. Such a value is in the order of magnitude of the ones published in references [51, 52] with microring biosensor based on the evanescent detection. To improve the detection limit in porous silica sensor, the design, and the choice of porosities will have to be yet optimized to get higher quality factor.

\section{Conclusion}

In this paper, porous silica ridge waveguides used in a MR structure have been designed, fabricated and characterized. The structure was created by photolithography of three different porous silica layers; the first one prevented resin infiltration during the photolithography process and the two others formed the core and the lower cladding of the porous ridge waveguide. The design of the MR was carried out with the aim of a future sensing application by defining a minimum detection wavelength range of $5 \mathrm{~nm}$ corresponding to a maximum shift of the resonant wavelength when molecules are attached in the pores of the porous structure. Knowing the refractive index of each porous layer, the dimensions of the ridge waveguide to provide single mode propagation were determined and a coupling study allowed us to determine the geometry of the racetrack resonator. Following this, porous silica ridge waveguide was obtained by plasma etching. SEM observations reveal that not only a top etching was obtained but there was also some lateral etching which reduced the width of the waveguide and increased somewhat the gap between the access waveguide and the racetrack resonator waveguide. Despite this, optical 
characterizations of the porous silica MR showed that it had been successfully implemented. Our current work, in progress, is to further optically characterize the resonator and the waveguides in order to determine the various losses. The MR based on porous silica ridge waveguides will be used as a sensor and we will experimentally determine its sensitivity which should be promising. This is because the molecules to be detected will infiltrate the porous silica core waveguide and will directly interact with the propagated light instead of with the evanescent waves in the MRs of the current literature. A very promising theoretical sensitivity $(1170 \mathrm{~nm} / \mathrm{RIU})$ of the porous racetrack MR has been obtained. It should be possible to improve this sensitivity by cascading several porous MRs [7].

\section{Acknowledgments}

This work is supported by the NORCAP and FOCUS projects, funded by the French Research Ministry, and the ARED funded by Conseil Général des Côtes d'Armor and the Région Bretagne.

\section{References}

[1] J. Charrier, C. Lupi, L. Haji, C. Boisrobert, Materials Science in Semiconductor Processing 3 (2000) $357-361$.

[2] P. Pirasteh, J. Charrier, A. Sotani, S. Haesaert, L. Haji, C. Godon, N. Errien, Applied Surface Science 253 (2006) $1999-2002$.

[3] R.L. Smith, S.F. Chuang and S.D. Collins, Sensors and Actuators A 21-23 (1990) 825 - 829.

[4] P. Steiner, F. Kozlowski, W. Lang, Appl. Phys. Lett. 62 (1993) 2700 - 2702. 
[5] M.G. Berger, R. Arens-Fischer, M. Thönissen, K. Krüger, S. Billat, H. Lüth, S. Hilbrich, W. Thei $\beta$, P. Grosse, Thin Film Solid 297 (1997) 237 - 240.

[6] S. Ilyas, J. Böcking, K. Kilian, PJ. Reece, J. Gooding, K. Gaus, M. Gal, Optical Materials 29 (2007) $619-622$.

[7] A. Foucaran, F. Pascal-Delannoy, A. Giani, A. Sackda, P. Combete, A. Boyer, Thin Film Solid 297 (1996) $317-320$.

[8] I. Rea, M. Iodice, G. Coppola, I. Rendina, A. Marino, L.D. Stefano, Sens. Actuators B: chem. 139, n¹ (2009) $39-43$.

[9] S. Chan, P.M. Fauchet, Y. Li, LJ. Roberg, BL. Miller, Phys. Status Solidi A- Appl. Res. 182 (2000) $541-546$.

[10] S. Dhanekar, S. Jain, Biosensors and bioelectronics 41 (2013) 54 - 64.

[11] S. M. Weiss, G. Rong, JL. Lawrie, Physica E 41 (2009) 1071 - 1075.

[12] X. Wei, S.M. Weiss, Optics Express 19, n¹2 (2011) 11330 - 11339.

[13] J. Lawrie, Z. Xu, G. Rong, P.E. Laibinis, S.M. Weiss, Phys. Status Solidi A 206, n6 (2009) $1339-1342$.

[14] A. Gharbi, B. Remaki, A. Halimaoui, D. Bensahel, A. Souifi, Semicond. Sci. Technol. 27 (2012) $105017-105022$.

[15] V. Yakovtseva, L. Dolgyi, N. Vorozov, N. Kazuchits, V. Bondarenko, Journal of Porous Material, 7 (2000) $215-222$.

[16] J.E. Lugo, J.A. Del Rio, J. J. Tagüeña-Martínez, J. Appl. Phys. 81 (1997) 1923 - 1928.

[17] N. Lorrain, M. Hiraoui, M. Guendouz, L. Haji, Material Science and Engineering B 176 (2011) $1047-1053$.

[18] V. Mulloni, L. Pavesi, Appl. Phys. Lett. 76 (2000) 2523 - 2525. 
[19] M. Hiraoui, L. Haji, M. Guendouz, N. Lorrain, A. Moadhen, M. Oueslati, Biosensors and Bioelectronics 36 (2012) $212-216$.

[20] J. Charrier, M. Guendouz, L. Haji, P. Joubert, Phys. Stat. Sol. (a) 182 (2000) 431 - 436.

[21] P. Pirastesh, J. Charrier, Y. Dumeige, S. Haesaert, P. Joubert, Journal of Applied Physics 101 (2007) 083110-1 - 083110-6.

[22] A. M. Rossi, G. Amato, V. Camarchia, L. Boarino, S. Borini, Appl. Phys. Lett. 78 (2001) $3003-3005$.

[23] K. Kim, T.E Murphy, Optics Express 21, n¹7 (2013) 19488 - 19497.

[24] M. Hiraoui, M. Guendouz, N. Lorrain, L. Haji, M. Oueslati, Appl. Phys. Lett. 101 (2012) 191114-1 - 191114-5.

[25] G.A Rodriguez, H. Shuren, S.M. Weiss, Optics Express 23, nº 6 (2015) 7111 - 7119.

[26] L. A. Delouise, P. M. Kou, B. L. Muller, Anal. Chem. 77 (2008) 3222 - 3230.

[27] W. Bogaerts, P. De Heyn, T. Van Vaerenberg, K. De Vos, S. Kumar Selvaraja, T. Claes, P. Dumon, P. Bienstman, D. Van Thourhout and R. Baets, Laser Photonics Rev. 6 (2012) 47 - 73.

[28] R. Landgraf, R. Kirchner, A. Finn, S. Arndt, T. Haugwitz, F. Deicke, W.J. Fischer, GMA/ITG-Fachtagung Sensoren und Messsysteme, Nürnberg 2012 Tagungsband (2012) 259 267.

[29] S. Soria, S. Berneschi, L. Lunelli, G. Nunzi Conti, L. Pasquardini, Advances in Science and Technology 82 (2011) $55-63$.

[30] A. Chiasera, Y. Dumeige, P. Féron, M. Ferrari, Y. Jestin, G. Nunzi Conti, Laser \& Photonics Reviews, 4, n³ (2010) $457-482$.

[31] F. Vollmer, S. Arnold, Nature methods, 5, nº7 (2008) $591-596$. 
[32] G.C. Righini, Y. Dumeige, P. Féron, M. Ferrari, G. Nunzi Conti, D. Ristic, Rivista del Nuovo Cimento 34, nº (2011), 435 - 488.

[33] C.Y. Chao, W. Fung, L.J. Guo, , IEEE journal of selected topics in quantum electronics, 12, $n^{\circ} 1(2006) 134-142$.

[34] C.Y. Chao, W. Fung, L.J. Guo, Journal of Lightwave Technology 24, n³ (2006) 1395 1402.

[35] M. Mancuso, J. M. Goddard, D. Erickson, Optics Express 20, n¹ (2012) 245 - 255.

[36] T. Hutter, N. Bamiedakis, S. R. Elliott, Proceedings of COMSOL Conference, (2010) Paris.

[37] D. Dai, Optics Express 17, n²6 (2009) 23817 - 23822.

[38] H. Yi, D. S. Citrin, Y. Chen, Z. Zhou, App. Phys. Lett., 95 (2009) 191112-1 - 191112-3.

[39] A. Ramachandran, S. Wang, J. Clarke, S.J. Ja, D. Goad, L. Wald, E.M. Flood, E. Knobbe, J.V. Hryniewicz, S.T Chu, D. Gill, W. Chen, O. King, B.E. Little, Biosensors and Bioelectronics 23 (2008) 939 - 944.

[40] J. Charrier, C. Lupy, L. Haji, C. Boisrobert, Materials Science in Semiconductor Processing $3(2000) 357-361$.

[41] K. Barla, R. Herino, G. Bomchil, J. Appl. Phys. 50 n² (1986) 439 - 443.

[42] M. Ivanda D. Ristic, , G. Speranza, Z. Siketic, I. Bogdanovic-Radovic, M. Marciuš, M. Ristic, O. Gamulin, S. Music, K. Furic, G. C. Righini, M. Ferrari, J. Phys. Chem. C 116 (2012) $1003910047-1003910050$.

[43] K. Okamoto, "Fundamentals of optical waveguides", 2nd edition, Academic Press (2006) p. $37-40,197-198$.

[44] E.A.J. Marcatili, Tech. J. 48 (1969) 2071 - 2102. 
[45] A. Maalouf, M. Gadonna, D. Bosc, Journal of Physics D: Appl. Phys. 42 (2009) 015106 -1 $-015106-11$.

[46] P. Girault, J. Lemaitre, M. Guendouz, N. Lorrain, L. Poffo, M. Gadonna, D. Bosc, SPIE Proceedings, Optical Sensing and Detection III 9141 (2014) 76 - 84.

[47] P. Girault, N. Lorrain, L. Poffo, M. Guendouz, J. Lemaitre, C. Carré, M. Gadonna, D. Bosc, G. Vignaud, J. Appl. Phys. 117, (2015) 104504-1 - 104504-8.

[48] M. Hiraoui, M. Guendouz, N. Lorrain, A. Moadhen, L. Haji, LM. Oueslati, Materials Chemistry and Physics 128 (2011) $151-156$.

[49] M. Dribek, J. Charrier, Journal of Applied Physics 107 (2010) 044905-1 - 044905-10.

[50] F. L. G. Flecha, V. Levi, Biochemistry and Molecular Biology Education 31, n5 (2003) $319-322$.

[51] A. Barrios, M. J. Bannls, V. Gonzales-Pedro, K.B. Gylfason, B. Sanchez, A. M. Holgado, R. Casquel, Optics Letters 33 (7) (2008) 708 - 710.

[52] M. K. Park, J. S/ Kee, J. Y. Quah, V. Netto, J. Song, Q. Fang, E. Mouchel La Fosse, G-Q Lo, Sensors and Actuators B 176 (2013) 552 - 559. 